Port Acadie

Revue interdisciplinaire en études acadiennes

An Interdisciplinary Review in Acadian Studies

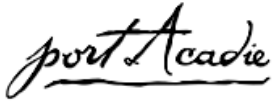

\title{
Les leçons à tirer de l'expérience de SOS-Églises
}

\section{David Tremblay}

Numéro 10-11-12, automne 2006, printemps-automne 2007

Le patrimoine religieux de la Nouvelle-Écosse : signes et paradoxes en Acadie

URI : https://id.erudit.org/iderudit/018645ar

DOI : https://doi.org/10.7202/018645ar

Aller au sommaire du numéro

Éditeur(s)

Université Sainte-Anne

ISSN

1498-7651 (imprimé)

1916-7334 (numérique)

Découvrir la revue

Citer cet article

Tremblay, D. (2006). Les leçons à tirer de l'expérience de SOS-Églises. Port Acadie, (10-11-12), 259-272. https://doi.org/10.7202/018645ar

\section{Résumé de l'article}

Que doivent faire les Acadiens pour s'assurer que leur patrimoine architectural religieux ne soit pas mis en péril par des décisions diocésaines de fermer et de détruire des églises paroissiales qui sont au coeur de leurs communautés? David Tremblay, stratège de la campagne menée par SOS-Églises dans le sud-ouest de l'Ontario pour sauver deux églises patrimoniales menacées de démolition, dégage de son expérience ce qu’i considère comme les éléments principaux d'un plan d'action efficace. 
TROISIÈME PARTIE : L'AVENIR DU PATRIMOINE RELIGIEUX

QUAND L'ÉGLISE DÉMISSIONNE

\section{Les leçons à tirer de l'expérience de SOS-Églises}

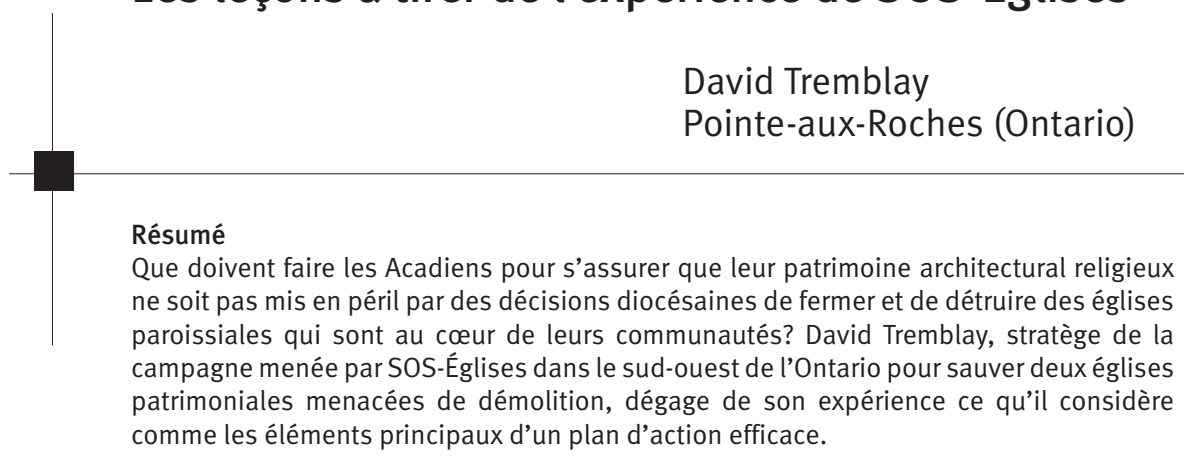

\section{Un mouvement de résistance}

$\mathrm{Au}$ nom des membres de SOS-Églises, je voudrais remercier très sincèrement les organisateurs de ce colloque de nous avoir donné cette occasion de vous faire part de l'expérience que nous vivons depuis plusieurs années dans le sud-ouest de l'Ontario.

Je voudrais tout d'abord vous demander d'être patient avec moi. Je vais vous parler dans une langue que vous aurez peut-être de la difficulté à comprendre parce que je parle avec l'accent de mon village. Je suis un Tremblay, c'est vrai, mais je ne suis pas un Tremblay du Lac Saint-Jean! Je suis David, fils de Paul, fils de Louis, fils d'Edgar, fils d'Edgar (père), fils de Jean-Baptiste de Pointe-aux-Roches. Je suis le fils de tous ces gens qui m'ont précédé. Quand nous pensons à qui nous sommes, il faut toujours se rappeler nos antécédents, parce que je crois que nous sommes sur cette terre pour continuer ce qui a été commencé par ceux et celles qui sont venus avant nous.

André Chénier vous a donné les grandes lignes de la lutte que nous menons pour empêcher la démolition de deux églises centenaires qui ont une grande valeur patrimoniale pour la communauté canadiennefrançaise de notre région. Mon rôle cet après-midi est de vous faire part de quelques réflexions qui se dégagent de l'expérience que nous avons vécue, afin d'aider d'autres communautés qui se retrouveront dans la même situation que nous.

La première chose que je voudrais vous dire au sujet de notre expérience, c'est que SOS-Églises n'est pas David Tremblay, ce n'est pas 
André Chénier. SOS-Églises n'est pas non plus une organisation structurée comme on l'entend d'habitude. SOS-Églises est plutôt un mouvement. Un mouvement, c'est quelque chose qui bouge, qui avance vers un objectif. Dans notre cas, la destination de notre mouvement, c'est d'empêcher la démolition de deux églises patrimoniales annoncée par le diocèse de London.

Il ne s'agit pas d'une résistance passive. Ce qui caractérise des mouvements comme SOS-Églises, c'est qu'ils tentent d'atteindre leur grand objectif par l'entremise de changements qu'ils essaient d'apporter au statu quo. SOS-Églises vit de changements. Même des changements inattendus. Heureusement pour nous, un grand nombre de ces changements, sinon la plupart, ont été positifs. Par exemple, si quelqu'un m'avait dit, il y a cinq ans, que j'irais en Nouvelle-Écosse, à l'Université Sainte-Anne, pour parler de nos églises, j'aurais eu beaucoup de difficulté à le croire. J'aurais pensé qu'il était impossible que ce dossier puisse jamais susciter suffisamment d'intérêt pour attirer l'attention des gens de si loin.

L'expérience de SOS-Églises prouve certainement que la sauvegarde de notre patrimoine culturel est un projet très difficile. Les gens de mon village me demandent souvent pourquoi j'ai entrepris un travail si exigeant. Pourquoi ne pas tout simplement accepter les décisions du diocèse? Après tout, il y a tellement d'autres bons projets avec de meilleures chances de réussite dans notre communauté.

Ma réponse est simple. Je m'oppose au projet du diocèse de démolir nos églises patrimoniales parce que ce projet est mauvais. II n'est pas important de savoir combien de personnes sont d'accord ou pas avec le projet. La destruction inutile du patrimoine d'une communauté est mauvaise. Quand un mal vient menacer ma communauté canadiennefrançaise et l'œuvre de mes ancêtres, je ne reste pas tranquille dans mon coin. J'essaye de faire ma part pour m'y opposer.

Je vous rappelle que le projet du diocèse de London est de démolir deux églises centenaires qui sont le cœur de deux villages canadiensfrançais, pour les remplacer, à deux fois le coût de leur réparation, par une nouvelle église construite à l'extérieur des villages, à quelques kilomètres d'un village très majoritairement anglophone.

Ce n'est pas important que les autorités religieuses aient organisé un référendum dont les résultats appuient le remplacement des églises. Quand une décision est vraiment mauvaise, elle reste mauvaise indépendamment du nombre de personnes que l'on a persuadées du contraire. Et tant que cette mauvaise décision n'a pas été exécutée, il n'est jamais trop tard pour essayer de la renverser. 
Il y a une chanson du folklore acadien qui m'a inspiré dans ma lutte pour sauver nos églises. Je connais cette chanson parce que l'amour de ma vie, mon épouse, donc mes enfants aussi, sont des Acadiens. Vous la connaissez tous, cette chanson :

Réveille! Réveille!

C'est les goddams qui viennent...!

Je dégage deux leçons de ces deux vers. Premièrement, il faut rester aux aguets. Deuxièmement, il faut comprendre que ces " goddams » qui menacent notre patrimoine peuvent être bien des choses. Des choses que l'on ne voit pas venir. Les "goddams » pourraient être les autorités religieuses qui veulent démolir nos églises pour servir leurs propres intérêts. Cela pourrait aussi être les pratiques commerciales qui nous amènent à toujours abandonner le vieux pour le remplacer par du neuf. Cela pourrait aussi être l'environnement naturel, quand de grands vents de la mer viennent secouer nos vieux bâtiments. Ces " goddams » pourraient être des tendances démographiques qui transforment nos villages en raison de migrations de populations urbaines vers la campagne. Pour pouvoir résister à tous ces "goddams ", à toutes ces menaces que l'on risque de ne pas voir venir, il faut tout d'abord être prêt. "Être prêt " peut vouloir dire bien des choses aussi. Je vais m'en tenir à trois grands thèmes : la planification, le regroupement et l'éducation.

\section{La planification}

Une communauté qui ne planifie pas la conservation de son patrimoine est destinée à ne plus avoir un jour que des souvenirs de son passé. On retrouvera peut-être des plaques ici et là nous informant de l'existence de tel ou tel bâtiment disparu. Mais le patrimoine lui-même n'existera plus que dans la mémoire de certaines personnes. Et ce patrimoine, avec la culture qu'il représente, finira par disparaître complètement.

Nous vivons à l'ère du visuel. Pour connaître et comprendre, les gens doivent voir. Il n'y a pas de meilleurs " raconteurs » de l'histoire d'une communauté que ses grands bâtiments impressionnants au cœur de nos villages. Ils sont des « raconteurs » extraordinaires parce qu'ils parlent toutes les langues et s'adressent à toutes les générations qui passent. Si nous perdons ces « raconteurs » de notre identité, nous disparaîtrons en tant que communauté culturelle, parce qu'une histoire n'existe que quand elle est racontée.

Dans ma région, qui est la plus ancienne communauté européenne à l'est de Montréal, il ne reste presque plus rien aujourd'hui qui rappelle nos trois cents ans d'existence. Rien d'autre que des papiers dans les 
archives. Des papiers que peu de personnes comprennent de toute façon, parce que la plupart sont écrits dans la langue de Cadillac. Notre histoire a besoin d'autres sortes de "raconteurs " que des documents écrits. Malheureusement, nous ne sommes pas les seuls dans cette situation. Au Canada, la planification de la sauvegarde des "raconteurs » de notre histoire devrait être un dossier prioritaire. Cette planification n'est pas quelque chose que l'on devrait faire demain. Ce n'est même pas quelque chose que l'on doit faire aujourd'hui. C'est quelque chose que l'on aurait dû faire hier. Cette obligation de planifier la protection de notre patrimoine doit tout d'abord être assumée par nos gouvernements municipaux, parce qu'ils sont les plus près des objets à conserver.

Si les « goddams » nous arrivent souvent par surprise, ne croyez pas cependant que leurs activités ne sont pas souvent planifiées longtemps d'avance. Ce n'est pas à la dernière minute que le diocèse de London a concocté son projet de démolition! Je ne serais pas surpris si ce projet de remplacement des églises actuelles était à l'état de planification depuis dix ans!

Cette partie de la communauté qui a à cœur l'avenir du fait français dans notre région n'avait pas malheureusement prévu une telle éventualité. Elle est donc arrivée un peu tard dans le dossier. Les décisions avaient déjà été prises au niveau de la planification diocésaine. L'expérience de SOS-Églises montre que, les diocèses n'étant pas des démocraties, il est bien difficile de faire renverser leurs décisions une fois qu'elles ont été prises. Tout au plus, les autorités religieuses peuvent accepter de nous écouter, mais elles n'ont aucune obligation de répondre à nos objections. Encore moins de changer leurs plans.

Heureusement, il y a d'autres avenues de résolution que SOS-Églises a explorées. La situation est bien différente dans les gouvernements municipaux, qui doivent fonctionner selon des principes démocratiques. Comme leurs membres doivent se faire élire, ces gouvernements sont beaucoup plus attentifs aux attentes et aux préoccupations des citoyens. C'est donc à ce niveau que SOS-Églises a investi beaucoup de ses efforts, tout en ne cessant pas d'essayer de convaincre les autorités religieuses de renverser leurs décisions.

Qu'est-ce que l'expérience de SOS-Églises nous a enseigné quant aux stratégies à planifier par rapport au secteur politique en préparation de la défense du patrimoine architectural? Elle nous a montré tout d'abord qu'il est très utile de connaître les rouages des conseils municipaux. Si la municipalité sait que vous connaissez bien son fonctionnement interne, surtout les lois et les règlements qui encadrent la planification et la prise de décisions, elle sera très prudente dans la façon dont elle dispose de vos réclamations. Le conseil municipal de Lakeshore a payé très cher en frais 
d'avocats sa tentative d'exclure SOS-Églises du débat en n'acceptant pas de recevoir sa proposition présentée en bonne et due forme concernant la désignation des églises.

Depuis ce temps, la possibilité d'une autre contestation judiciaire inquiète le conseil. Les membres du conseil savent que nous conservons nos liens avec maître Caza. Et nos multiples appels à la Commission des affaires municipales de la province leur rappellent qu'ils doivent faire les choses correctement et ne pas se laisser trop influencer par les pressions exercées par de grandes institutions, comme le diocèse. Les défenseurs du patrimoine doivent aussi bien s'informer des obligations municipales en matière de protection du patrimoine. Ces obligations émanent de lois provinciales; alors, il faut aussi bien connaître la législation propre à ce palier de gouvernement. Mais attention! Il ne faut pas se laisser endormir par de belles phrases réconfortantes qui contiennent des mots comme " peut », "peuvent » et « pourraient». Sachez que «pourrait » peut aussi vouloir dire «ne pourrait pas »! Une bonne planification veut dire que l'on s'assure que les lois nous protègent bien avant que nous ayons besoin de leur protection.

Une partie de la planification à long terme pour la protection du patrimoine se fait donc par l'entremise de pressions à exercer auprès des gouvernements pour les amener à changer leurs lois quand elles sont inadéquates ou à améliorer l'application des lois existantes, le cas échéant. Il faut pousser les gouvernements à faire de la protection de notre patrimoine une question d'obligation et non de choix. En passant, en Ontario, il est évident que l'impact de SOS-Églises a été un facteur déterminant dans la récente décision du gouvernement de renforcer la loi sur le patrimoine, pour faire en sorte qu'un bâtiment désigné par une municipalité comme site patrimonial ne puisse plus être démoli, comme c'était le cas auparavant.

Dans la planification, il ne faut pas non plus chercher à éviter ou à contourner toutes les situations difficiles. SOS-Églises a planifié des actions audacieuses. Par exemple, il y a eu l'organisation de ralliements avec le risque que personne ne s'y présente. SOS-Églises a organisé un ralliement qui s'est déroulé à l'heure même de la dernière partie pour la médaille d'or au hockey mettant aux prises le Canada et les États-Unis, en 2002. Malgré cela, plus de 200 personnes ont accepté l'invitation de se rendre dans une grande salle pour écouter des discours durant cette partie. Ce simple fait a été en soi une preuve importante du niveau d'appui populaire que reçoivent les efforts pour la conservation de nos églises.

Comme entreprise audacieuse, il y a aussi eu l'embauche, à grands risques financiers, d'avocats chevronnés et dispendieux, sans même avoir l'assurance que leur intervention donnerait les résultats escomptés. 
Quand nous avons approché maître Ronald Caza - l'avocat du dossier de l'Hôpital Montfort - pour faire suspendre par voie d'injonction la démolition de l'église de Saint-Joachim, il m'a rappelé qu'une intervention judiciaire au niveau de la Cour supérieure allait inévitablement coûter de gros sous. Il nous a aussi averti que nos chances de succès étaient minces, parce que les cours de justice n'arrêtent que rarement par injonction des projets de démolition qui ont déjà commencé. Et, de plus, insister comme nous le faisions pour que cette démarche judiciaire se fasse en français à Windsor rendait le défi encore plus difficile. Mais nous avons persisté et les résultats ont été positifs. Une demande d'aide financière à la communauté nous a rapporté 50000 dollars dans l'espace de deux semaines. Nous avons gagné notre cause en cour et le perdant - la municipalité - a été forcé de payer le gros de nos dépenses d'avocats. Le reste a été payé par le Fonds de contestation judiciaire du fédéral (ill. 1, 2, 3, 4).

\section{Le regroupement}

Je voudrais maintenant vous parler d'un autre thème : le regroupement des forces en faveur de la protection du patrimoine. Une des leçons qui se dégagent de l'expérience de SOS-Églises est que les gens s'intéressent à la protection du patrimoine pour toute une gamme de raisons. Pour certains, un bâtiment doit être conservé pour des raisons religieuses. Pour d'autres, les raisons seront culturelles ou communautaires. D'autres encore verront dans le bâtiment un intérêt architectural qui justifie sa conservation. Les gens d'affaires considéreront qu'il est important de ne pas enlever à un village son cœur architectural, à cause des répercussions que cela entraînerait sur le chiffre d'affaires des commerces ou sur la valeur immobilière des autres propriétés. Les membres de l'industrie du tourisme voudront aussi empêcher la perte de sites d'intérêt touristique. Les écologistes seront aussi de la partie. Ils verront mal que l'on envoie au dépotoir des édifices qui ont encore de nombreuses années de service à rendre à la communauté.

Le défi d'un regroupement comme SOS-Églises est de rassembler dans une même action toutes ces parties aux intérêts différents. Non seulement différents, mais quelquefois en conflit. Celui qui veut conserver le bâtiment comme lieu de culte verra d'un mauvais oeil le projet d'un autre qui voudra exploiter la valeur commerciale de l'édifice.

Nous avons assez bien réussi à éviter ces conflits dans notre mouvement, en nous entendant sur une façon de procéder et en nous concentrant sur un seul grand objectif. La façon de procéder : allons-y une étape à la fois. L'objectif : que les édifices restent debout. C'est dans un deuxième temps que viendra le moment de prendre position par rapport aux diverses options sur la table. Les gens ont tous compris et accepté 


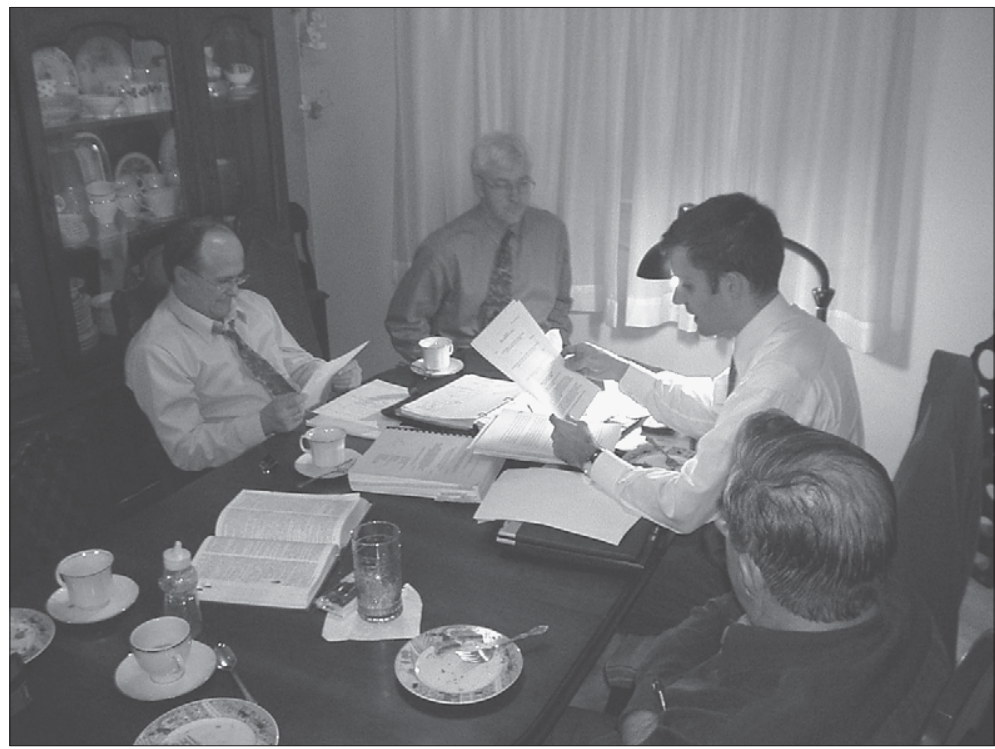

1. SOS-Églises : David Tremblay, porte-parole de SOS-Églises, en compagnie des avocats Ronald Caza et Rodrigue Escayola, de renommée nationale depuis le dossier SOSMontfort.

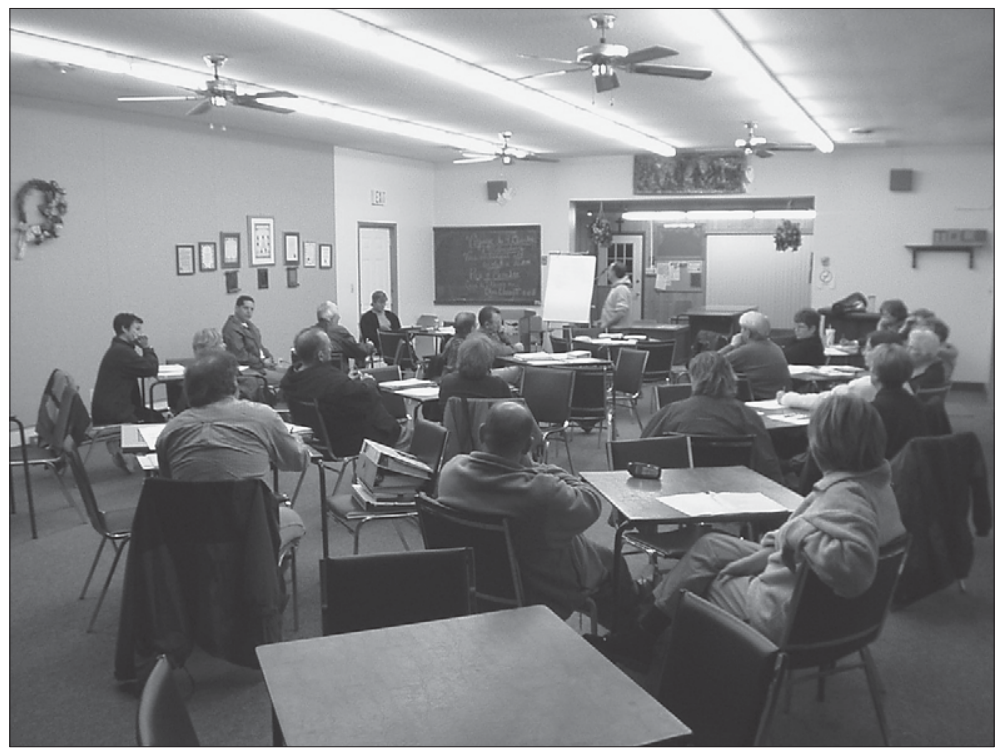

2. SOS-Églises : une des nombreuses réunions des membres du collectif. 


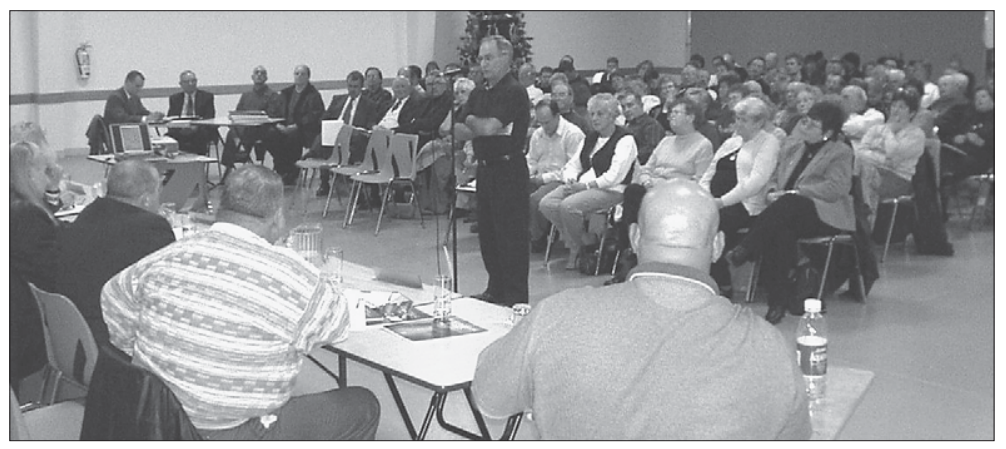

3. SOS-Églises : une des nombreuses interventions auprès du Conseil municipal de Lakeshore.

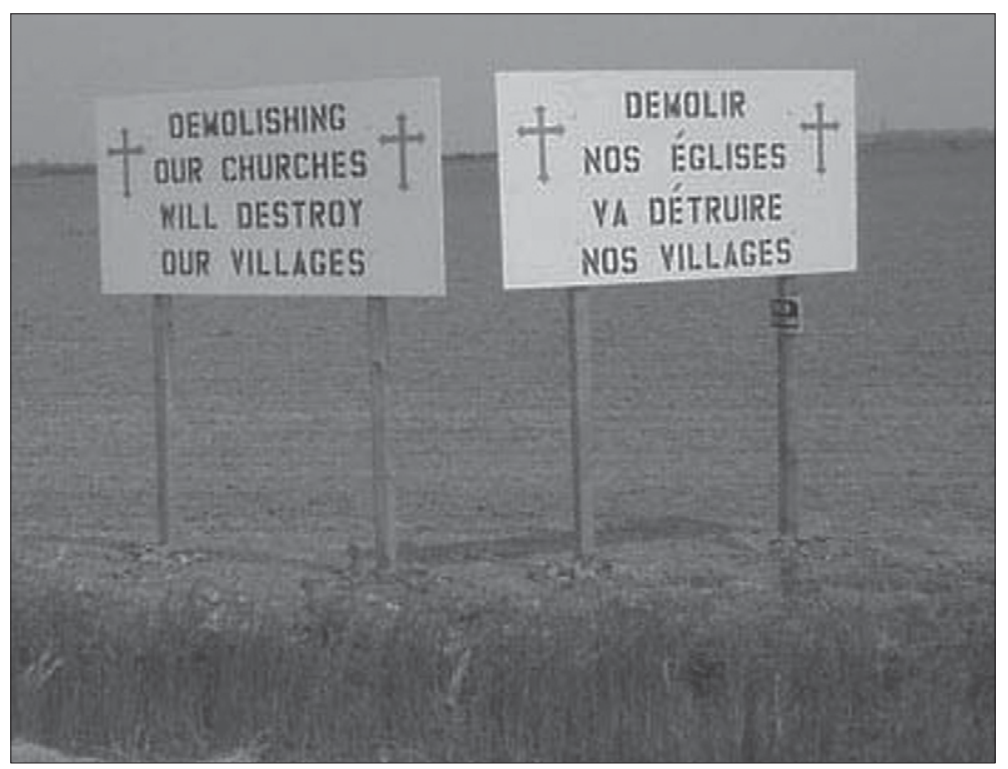

4. «Démolir nos églises va détruire nos villages », panneaux d'information bilingues installés à l'entrée du village de Saint-Joachim. Des panneaux semblables ont aussi été installés à Pointe-aux-Roches. 
que, si les églises passaient sous le pic du démolisseur, leurs aspirations n'auraient qu'un intérêt théorique! Pour assurer la solidarité du groupe, nous n'avons pas hésité à faire appel à deux sentiments que nous avions tous en commun : notre attachement aux deux églises et la peur de les perdre.

Nous n'avons jamais fait de campagne de recrutement pour notre groupe de travail. Les quelque trente personnes qui assistent régulièrement à nos réunions d'affaires - toutes les deux semaines pendant plusieurs années - se présentent de leur propre gré sans pression aucune. Les circonstances ont fait que notre groupe est formé de personnes très variées, aux talents très différents. Nous avons une personne experte en architecture. Nous avons un ancien gérant de banque qui analyse toutes les données financières émanant du diocèse. Récemment, un jeune avocat s'est joint au groupe. Depuis le tout début, nous avons été enrichis par la présence de paroissiens et paroissiennes dont les convictions religieuses sont profondes et bien connues. Comme on vous l'a déjà dit, j'ai beaucoup d'expérience en politique municipale. En plus des Canadiens français, nous comptons des Canadiens anglais ainsi que plusieurs Américains résidant au Canada. Certains de nos membres ont le tempérament fougueux et nous encouragent à brasser la cage plus fortement. D'autres prêchent la patience et la discipline. Plusieurs se considèrent même comme des amis personnels de ceux et celles qui proposent la démolition des églises.

Pour être efficace, il ne suffit pas qu'un groupe ait des membres convaincus et compétents. Il lui faut aussi des outils. L'outil principal dont SOS-Églises s'est servi, cela a été les médias. Il faut reconnaître, et c'est tout à leur honneur, que les médias ont perçu dès le tout début l'importance du dossier. Un des premiers gestes posés en opposition aux plans de démolition du diocèse a été de distribuer un simple message dans la communauté pour inviter les intéressés à un rassemblement. À notre surprise, plus de 350 personnes se sont présentées à la réunion! Et ce qu'il y a eu de merveilleux, c'est que des journalistes de deux postes de télévision sont venus nous entendre. Le résultat a été que l'essentiel de ce que nous avons présenté aux personnes qui ont répondu à notre invitation a été diffusé partout dans la région dans les journaux et à la télévision. Une cause publique venait de naître.

Il fallait assurer un lendemain à cet intérêt initial. Nous avons reconnu dès le début que notre réputation comme mouvement sérieux dépendait autant de notre style de fonctionnement que de nos activités. Il était évident que les médias ne nous accorderaient pas beaucoup d'attention si nous étions perçus tout simplement comme un groupe de mécontents en chicane avec le curé ou l'évêque. 
Nos différends avec les autorités religieuses n’ont jamais eu rien de personnel et nous faisons tous les efforts nécessaires pour le prouver. Par exemple, à titre de président, je ne permets à aucun membre durant nos réunions de dire des choses diffamatoires concernant nos adversaires. Je suis convaincu que, si nous nous étions laissés aller à de telles émotions, nous aurions vite perdu plusieurs membres importants de notre groupe en plus du respect de la population et des médias. D’autre part, si les groupes qui luttent pour sauvegarder leur patrimoine religieux sont perçus comme des ennemis du curé, ils perdront l'appui de tous les membres des Chevaliers de Colomb et des Dames de Sainte-Anne, ainsi que de tous les parents qui veulent un jour faire baptiser leurs nouveau-nés. SOS-Églises se limite à disséminer des informations sur la situation et sur les enjeux, tout en évitant de porter des jugements sur les motivations ou les valeurs personnelles de nos adversaires.

La transparence caractérise aussi notre style de fonctionnement. Non seulement nous sommes toujours disponibles pour des entrevues, mais nous acceptons même que les journalistes assistent à nos réunions s’ils le désirent. Nos procès-verbaux sont à leur disposition. En ne cachant rien et en fonctionnant au grand jour, nous avons obtenu que les médias nous maintiennent la confiance qu'ils avaient eue dès le départ en notre intégrité. Ils nous croient quand nous disons que nous sommes motivés par l'amour de notre patrimoine et non par des sentiments vindicatifs, ou même par un désir de résister au changement. Ils savent que nos intérêts ne sont pas personnels et que nous visons le bien-être de la communauté.

L'implication des médias dans notre dossier nous a rendu service de bien des façons. Quand j'ai été accusé d'être entré par effraction dans l'église de Saint-Joachim pour sonner la cloche pendant un de nos ralliements, ce sont les médias qui ont informé la police qu'ils avaient sur vidéo la preuve que cela ne pouvait être moi, parce que j'étais sur le parvis de l'église pendant que la cloche sonnait. Soit dit en passant, le diocèse n'a pas redoré son image médiatique en demandant à la police de déposer une charge criminelle contre moi. Ma photographie est parue sur la première page du Windsor Star, accompagnée d'un article satirique disant que j'étais accusé du crime d'avoir sonné la cloche de l'église de Saint-Joachim! Nous avons profité de cette couverture médiatique pour rappeler à la population que l'église qui était destinée à la démolition avait été abandonnée sans tenir compte des exigences du droit canon par rapport à la fermeture d'une église. Si nous étions coupables de ce terrible crime d'avoir sonné la cloche, notre action aurait eu le mérite de rendre un dernier hommage à ce vieil ami de la communauté avant sa démolition, en lui permettant de faire entendre sa voix une dernière fois 
dans le village. Nous ne comprenions vraiment pas le diocèse, qui voyait là matière à poursuite criminelle! Sur le plan stratégique, le diocèse a fait l'erreur, dans cet incident, de confondre son antagonisme envers un individu, ma personne, avec son objection à notre campagne. Ce geste a été loin d'aider sa cause aux yeux du grand public.

Ma présence ici est aussi le résultat de la couverture que nous avons reçue des médias. Sans leur intervention, jamais notre cause n'aurait été connue sur le plan provincial, ensuite sur le plan national pour enfin atteindre même l'Acadie! À cause de cette couverture médiatique continue, qui s'est manifestée depuis les tout débuts de notre campagne, nous avons commencé à recevoir de l'appui de toutes sortes de sources importantes. Par exemple, le commissaire aux langues officielles, Dyane Adam, suit notre dossier de près. Elle a même affecté une avocate de son bureau pour nous aider dans notre contestation judiciaire.

Nous pensons que notre action a contribué à faire en sorte que l'opinion publique est de plus en plus en faveur de la conservation du patrimoine. Il y a quelques semaines, un poste de radio anglophone très populaire a effectué un sondage téléphonique sur l'importance de sauver l'église de Saint-Joachim. Des 500 auditeurs qui ont participé, 75 pour cent se sont prononcés en faveur de la sauvegarde de l'église. Il n'y a pas eu de concertation des votes de notre part. Nous ne savions même pas que ce sondage allait avoir lieu. Sans les médias, jamais nous n'aurions pu recevoir un tel appui populaire.

\section{L'éducation}

Nous avons beaucoup de travail à faire pour sensibiliser les gens à l'importance du patrimoine. Surtout nos bâtiments. Comme je l'ai dit plus haut, nos édifices patrimoniaux sont les meilleurs "raconteurs » de notre histoire. Ils sont donc les meilleurs témoins de notre identité culturelle. Il faut répandre ce message à tous les niveaux : le conseil paroissial, le conseil municipal, le gouvernement provincial.

J'ai déjà suffisamment insisté sur l'importance que nous avons accordée à la participation des médias au dossier. Nous avons passé des heures au téléphone avec des journalistes pour leur donner tous les renseignements dont ils avaient besoin pour bien interpréter les événements. Nous ne prenions pas pour acquis que les journalistes avaient la science infuse et connaissaient à priori et à fond tous les dossiers qu'on leur demandait de couvrir. Ces journalistes nous étaient très reconnaissants de notre disponibilité. Ils nous disaient que nos adversaires, eux, étaient très difficiles à rejoindre pour des explications de leur point de vue. Souvent, c'est nous qui avions à essayer de leur expliquer le comportement et les décisions de nos adversaires! 
SOS-Églises a tenté d'informer la communauté de l'importance de la conservation du patrimoine principalement au moyen des activités suivantes : 1) des ralliements au cours desquels des invités de marque sont venus exprimer leur appui et expliquer pourquoi nos efforts étaient justifiés; 2) de multiples entrevues à la radio et à la télévision, et avec la presse écrite; 3) des bulletins périodiques adressés à tous les foyers des trois villages. Présentés dans les deux langues, ces bulletins expliquent les raisons pour lesquelles il ne faut pas que le diocèse donne suite à son projet de démolition des églises. Tous les foyers reçoivent ces messages, autant les catholiques que les non-catholiques parce que nous estimons que la protection du patrimoine religieux est l'affaire de tout le monde, indépendamment des appartenances paroissiales; 4) des délégations et présentations auprès du conseil municipal sur divers sujets relatifs à la protection des églises; 5) du porte-à-porte pendant la dernière campagne électorale municipale pour sensibiliser l'électorat à l'importance de la conservation du patrimoine architectural dans la planification municipale. Je me suis moi-même présenté comme candidat à la mairie et j'ai forcé mes adversaires à prendre position par rapport au rôle de la municipalité à l'égard de la conservation du patrimoine. Évidemment, tous ont dû se dire en faveur. Ils doivent maintenant être fidèles à cette position, ou du moins ne pas la contredire carrément!

À tout ceci s'est ajoutée la contribution d'un jeune metteur en scène, qui a écrit une pièce de théâtre dans laquelle les comédiens et comédiennes interprétaient des personnages qui représentaient les divers points de vue sur ce dossier. La teneur générale de la pièce était évidemment en faveur de la cause de SOS-Églises. C'est un des membres de SOS-Églises qui a conçu et fabriqué le décor, qui était une reproduction du clocher de l'église de Saint-Joachim que les personnages construisaient tout au cours la pièce.

Dans nos ralliements, entrevues médiatiques etautres manifestations, nous avons profité de chaque occasion pour répondre aux questions ou objections à la conservation de bâtiments patrimoniaux, des objections comme : "Cela coûtera trop cher de réparer ces églises »; ou bien : "Le genre de matériau requis pour restaurer les églises n'existe plus »; ou bien : "Les gens sont trop occupés de nos jours pour entreprendre des projets comme ça »; ou bien encore : "Il n'y a plus personne dans la communauté qui saurait comment faire »; ou bien : "Nous sommes une trop petite communauté pour faire cela », etc. Nous avons rappelé aux gens qu'il y avait des réponses à toutes ces objections. La génération d'aujourd'hui n'est pas moins habile, moins bien équipée, moins libre que nos ancêtres qui ont su surmonter eux aussi toutes sortes de difficultés 
beaucoup plus grandes pour nous laisser ce patrimoine. Soyons francs : c'est une question de priorité, pas de moyens.

\section{Conclusion}

Je vous ai présenté dans ces pages un aperçu de quelques expériences que nous avons vécues à SOS-Églises. Ce qu'André Chénier et moi-même vous avons dit n'est pas l'histoire complète de notre campagne, mais nous espérons que vous y avez trouvé des renseignements utiles.

Je termine cet exposé en énumérant des faits qui montrent l'impact qu'a eu SOS-Églises, directement ou indirectement, depuis son établissement il y a cinq ans. 1) Les églises sont encore là. 2) La valeur patrimoniale des deux églises a été professionnellement confirmée et établie par une firme d'architectes indépendants. 3) Il est probable que la municipalité donnera, par la voie de la désignation patrimoniale, la possibilité à la communauté de trouver une autre vocation à l'église de Pointe-aux-Roches. Il y a toujours une possibilité que la municipalité décide d'accorder pleine désignation à l'église de Saint-Joachim après les audiences auprès de la Commission des biens publics à l'automne. 4) La conservation du patrimoine est devenue un sujet d'actualité dans la municipalité et reçoit l'appui du public. 5) Le conseil municipal est en train d'établir un comité sur le patrimoine, ce qu'il avait toujours refusé de faire. Le chef de SOS-Églises y siégera. 6) La majorité des membres du conseil se sont prononcés en faveur de la conservation du patrimoine en général, et de la protection totale ou partielle des deux églises. 7) Le diocèse de London s'est rendu compte que la communauté n'est pas seulement un témoin impuissant devant ses agissements. 8) Un mémoire a été présenté au diocèse de London sur la mise en œuvre de son programme de gestion d'édifices religieux excédentaires. 9) Un mémoire a été présenté au cardinal Turcotte de Montréal pour l'inciter à proposer l'établissement de mesures à prendre sur le plan national pour éviter une situation comme celle que nous avons connue dans le sud-ouest ontarien. 10) Le besoin de renforcer la loi ontarienne sur le patrimoine a été mis en évidence. Cette loi a été révisée l'an passé. 11) Une solidarité plus forte s'est développée entre les membres de la communauté canadienne-française locale. 12) La présence et la vitalité de la communauté canadienne-française du sudouest de l'Ontario ont été affirmées publiquement sur le plan régional, provincial et national. 13) La communauté canadienne-française d'ici et d'ailleurs est davantage alertée au danger qui menace son patrimoine religieux.

En conclusion, je voudrais vous remercier encore de m'avoir donné l'occasion de venir vous rencontrer. Comme je vous ai dit, mon épouse est acadienne, donc mes enfants le sont aussi. C'est donc le patrimoine de 
mes enfants que je vous demande de protéger dans vos milieux acadiens. C'est du fond du cœur que je vous souhaite le plus grand succès. 